\title{
Using network analysis to examine links between individual depressive symptoms, inflammatory markers, and covariates
}

\author{
Supplementary materials at: \\ https://osf.io/5832e/
}

\author{
Fried, E. I. ${ }^{1 *}$ \\ von Stockert, S. ${ }^{2}$ \\ Haslbeck, J. M. B. ${ }^{2}$ \\ Lamers, F. ${ }^{3}$ \\ Schoevers, R. A. ${ }^{4}$ \\ Penninx, B. W. J. H. ${ }^{5}$
}

${ }^{1}$ Department of Clinical Psychology, Leiden University, Leiden, The Netherlands.

${ }^{2}$ Department of Psychology, University of Amsterdam, Amsterdam, The Netherlands.

${ }^{3}$ Amsterdam UMC, Vrije Universiteit, Psychiatry, Amsterdam Public Health research institute, The Netherlands.

${ }^{4}$ Department of Psychiatry, Interdisciplinary Center Psychopathology and Emotion Regulation (ICPE), University Medical Center Groningen, University of Groningen, Groningen, The Netherlands.

${ }^{5}$ Amsterdam UMC, Vrije Universiteit, Department of Psychiatry and Neuroscience Campus Amsterdam, Amsterdam Public Health research institute, The Netherlands.

Corresponding author: Eiko Fried, eikofried@gmail.com. Kloosterstraat 55b, 2021VK Haarlem, The Netherlands.

Financial support: The infrastructure for the NESDA study (www.nesda.nl) is funded through the Geestkracht program of the Netherlands Organisation for Health Research and Development (ZonMw, grant number 10-000-1002) and financial contributions by participating universities and mental health care organizations (VU University Medical Center, GGZ inGeest, Leiden University Medical Center, Leiden University, GGZ Rivierduinen, University Medical Center Groningen, University of Groningen, Lentis, GGZ Friesland, GGZ Drenthe, Rob Giel Onderzoekscentrum). Assaying of inflammatory markers was supported by the Neuroscience Campus Amsterdam. During writing this paper, EIF was in part funded by the ERC Consolidator Grant no. 647209.

Word count: 4431 
Background. Studies investigating the link between depressive symptoms and inflammation have yielded inconsistent results, which may be due to two factors. First, studies differed regarding the specific inflammatory markers studied and covariates accounted for. Second, specific depressive symptoms may be differentially related to inflammation. We address both challenges using network psychometrics.

Methods. We estimated seven regularized mixed graphical models in the NESDA data $(\mathrm{N}=2321)$ to explore shared variances among 1) depression severity, modeled via depression sum-score, 9 DSM-5 symptoms, or 28 individual depressive symptoms; 2) inflammatory markers C-reactive protein (CRP), interleukin 6 (IL-6), and tumor necrosis factor $\alpha(\mathrm{TNF}-\alpha)$; 3) before and after adjusting for sex, age, body mass index (BMI), exercise, smoking, alcohol, and chronic diseases.

Results. The depression sum-score was related to both IL-6 and CRP before, and only to IL-6 after covariate adjustment. When modeling the DSM-5 symptoms and CRP in a conceptual replication of Jokela et al. (2016), CRP was associated with 'sleep problems', 'energy level', and 'weight/appetite changes'; only the first two links survived covariate adjustment. In a conservative model with all 38 variables, symptoms and markers were unrelated. Following recent psychometric work, we re-estimated the full model without regularization: the depressive symptoms 'insomnia, 'hypersomnia', and 'aches and pain' showed unique positive relations to all inflammatory markers.

Conclusions. We found evidence for differential relations between markers, depressive symptoms, and covariates. Associations between symptoms and markers were attenuated after covariate adjustment; BMI and sex consistently showed strong relations with inflammatory markers.

Keywords: depression, inflammation, individual depressive symptoms, network analysis, body mass index 


\section{Introduction}

Major Depressive Disorder (MDD) is a debilitating condition that is associated with a considerable reduction in quality of life, functional disability, and social impairment (Dunn 2012; Bockting et al. 2015). In the search for etiological factors that explain the development of MDD, systemic low-grade inflammation has been suggested as a candidate mechanism. Sickness behavior-related symptoms such as fatigue, loss of energy, motor slowing, or social withdrawal resemble symptoms that are associated with depression (Dantzer et al. 2008). It has therefore been proposed that depression may constitute a maladaptive or exacerbated form of sickness behavior occurring in cases where inflammation is permanent and systemic (Smith 1991; Dantzer et al. 2008; Haroon et al. 2012).

Several recent meta-analyses examining cross-sectional and prospective links between depressive symptoms or MDD on the one hand, and inflammatory markers on the other, have either yielded small effects (Valkanova et al. 2013; Haapakoski et al. 2015a; Köhler et al. 2017; Horn et al. 2018; Smith et al. 2018), or no effects in a selection of higher quality studies (Horn et al. 2018). Effect sizes for specific inflammatory markers such as C-reactive protein (CRP), interleukin-6 (IL-6), and tumor necrosis factor alpha (TNF- $\alpha$ ) show considerable heterogeneity, both across individual studies and individual metaanalyses. These findings indicate remaining uncertainty regarding the relationship between depression and inflammation. They also highlight the important role of demographic, lifestyle, or disease-related covariates, since relations are often attenuated or disappear completely when additional factors are considered (O'Connor et al. 2009; Smith et al. 2018).

We see three reasons for the inconsistency in the literature that we aim to address in the present study. First, studies differed regarding the specific inflammatory markers studied. While a detailed review of the literature is beyond the scope of the present paper, it is worth noting that different markers serve different biological functions and are not interchangeable. CRP and IL-6, for instance, are increasingly understood as markers of somatic maintenance rather than an acute inflammatory response, contrasting TNF- $\alpha$ (Del Giudice \& Gangestad 2018).

Second, studies differed regarding covariates accounted, which might in part explain differences in results (O'Connor et al. 2009; Köhler et al. 2017; Smith et al. 2018). This calls for work that includes several inflammatory markers; includes more covariates; and examines relations between depression and inflammatory markers before and after covariate adjustment.

Third, most previous studies investigated the link between inflammatory markers and a sum of depressive symptoms or MDD diagnoses, which leaves open the possibility that links between markers and symptoms occur differentially. If only a subset of symptoms is related to inflammatory markers, sum scores or diagnoses would lack power to pick up associations. Differential relations are plausible given recent work in the emerging field Symptomics, showing that individual depressive symptoms differ in their associations with risk factors (Fried et al. 2014), neural activity (Stringaris et al. 2015), 
impairment of functioning (Tweed 1993; Fried \& Nesse 2014), in response to life events (Keller et al. 2007), and in response to antidepressant treatment (Hieronymus et al. 2016) (see Fried \& Nesse 2015 for a review). In sum, differential relations between depressive symptoms and inflammatory markers could explain inconsistent results in the literature, and offer an opportunity to move the field forward.

Seven studies provide preliminary evidence for such differential relations between depressive symptoms and inflammation. Jokela et al. (2016) modeled associations between nine DSM-5 symptoms and CRP, and identified robust relations of CRP with sleep problems, tiredness, and changes in appetite. Fairly consistent with this study, White et al. (2017) found specific relations between CRP and restless sleep, fatigue, low energy, and feeling depressed. Lamers et al. (2017) identified robust associations between the MDD symptom increased appetite and markers CRP and TNF- $\alpha$. Chu et al. (2018) found that IL-6, but not CRP levels at age 9 predicted diurnal mood variation, concentration difficulties, fatigue, and sleep disturbances at age 18; somatic and psychological symptom dimensions were related to IL-6, but not CRP. Duivis et al. (2013) split depressive symptoms into a cognitive and a somatic subscale, and found associations between somatic symptoms and CRP, IL- 6 and TNF- $\alpha$; the relationships disappeared when covariates were controlled for. Moriarity et al. (2019) examined a prospective cohort of adolescents and investigated predictive relationships of baseline inflammatory markers on changes in 5 depression subscales. CRP levels - but not IL-6, IL-8, IL-10, or TNF- $\alpha$ levels-predicted increases in the lack of personal and social interest subscale, controlling for several demographic and biological variables. Finally, Lamers et al. (2016) identified differences between the relation of melancholic and atypical depression subtypes to inflammatory markers, which could be driven by differences between individual symptoms.

In the present study, we explore links between the markers CRP, IL-6 and TNF- $\alpha$ and depressive symptoms with and without covariate adjustment in a large diverse sample of 2321 participants. In doing so, we extend the prior literature in seven aspects. First, some prior studies decomposed the depression sum-score into subtypes or subscales — often with limited reliability (Moriarity et al., 2019) — but did not model individual symptoms. Second, studies that modeled individual symptoms focused on a limited subset of symptoms such as the DSM-5 criteria (Jokela et al. 2016) or the 8-item CES-D (White et al. 2017). However, DSM descriptions are narrower than descriptions of MDD found in textbook literature (Kendler 2016), and common rating scales for depression feature over 50 distinct symptoms (Fried, 2017b). To maximize content validity, we chose to model 28 depressive symptoms. Third, most studies featured a limited set of inflammatory markers and covariates, which we extend in the present study to three markers and seven covariates. Fourth, we utilize a large sample of 2321 participants along the whole continuum of depression severity. Fifth, we use network models specifically developed for uncovering unique shared associations in highly multivariate data (Epskamp \& Fried 2018). The goal is to test whether specific symptoms of depression are related to specific inflammatory markers after controlling for all other depressive symptoms, markers, and covariates. Sixth, we provide the first 
conceptual replication of a prior study on depressive symptoms and inflammatory markers (Jokela et al. 2016), using the same variables. Finally, we model the relationships between markers and symptoms in various stages of complexity, from networks with 4 nodes to networks of 38 nodes, and specifically investigate the impact covariate adjustment has on the relationships. Given the inconsistency of the prior literature, we have no strong a priori hypotheses; the nature of the paper is exploratory.

\section{Method}

\section{Participants}

The present study used data gathered as part of the Netherlands Study of Depression and Anxiety (NESDA), a multisite, naturalistic, longitudinal cohort study that observes the course of mood and anxiety disorders (for details, see Penninx et al. 2008). Participants in this study were recruited from Dutch primary care practices, specialized mental health institutions, as well as from community samples. The total sample comprises 2981 participants: 373 healthy subjects, 1701 participants with a current depressive and/or anxiety disorder, and 907 participants with earlier episodes of these disorders or at high risk for their development. We included all 2321 participants who had no missing data, covering the whole continuum of depressive symptomatology from healthy to severe clinical depression. We excluded 122 participants with CRP levels above $10 \mathrm{mg} / \mathrm{L}$ to avoid bias due to acute infection; a Welch Two Sample t-test revealed that these participants $(\mathrm{M}=22.67, \mathrm{SD}=12.58, \mathrm{~N}=122)$ did not differ from the analytic sample $(\mathrm{M}=20.85, \mathrm{SD}=14.1, \mathrm{~N}=2321)$ on the Inventory of Depressive Symptomatology, $t(137.48)=1.55, p=0.123$. The Ethical Commission of each participating care center approved the study protocol. All participants provided written informed consent.

\section{Measurement Instruments}

\section{Inventory of Depressive Symptomatology (IDS)}

NESDA used the self-rated version of the Inventory of Depressive Symptomatology to assess depression severity (IDS-SR; Rush, Gullion, Basco, Jarrett, \& Trivedi, 1996); see Table 1. Symptoms were scored $0-3$ and rated regarding frequency in the last week before assessment. We analyzed 28 symptoms, 9 DSM-5 MDD criteria, and the sum-score. Symptoms 11 and 12 on the IDS constitute compound measures that assess appetite increase/decrease and weight increase/decrease; unfortunately, their coding scheme did not allow us to separate increase from decrease. To estimate scores on DSM-5 MDD criteria, individual IDS symptoms were compounded by their maximum value (e.g. sleep problems was coded as the highest score on any of the four sleep-related items). 
Table 1. IDS Symptoms

\begin{tabular}{lll}
\hline IDS Symptoms & Mean & SD \\
\hline 1 Early Insomnia & 1.84 & 1.06 \\
2 Mid Insomnia & 2.27 & 1.05 \\
3 Late Insomnia & 1.49 & 0.87 \\
4 Hypersomnia & 1.47 & 0.70 \\
5 Sad Mood & 1.83 & 0.86 \\
6 Irritable Mood & 1.87 & 0.82 \\
7 Anxious Mood & 1.91 & 0.85 \\
8 Mood Reactivity & 1.44 & 0.74 \\
9 Mood Variation & 1.48 & 0.82 \\
10 Mood Quality & 1.84 & 1.07 \\
11 Appetite Decrease/Increase & 1.62 & 0.89 \\
12 Weight Decrease/Increase & 1.74 & 0.94 \\
13 Concentration/Decision making & 1.84 & 0.86 \\
14 Self-blame/Worthlessness & 1.92 & 1.18 \\
15 Outlook on the Future & 1.83 & 0.75 \\
16 Suicidal Ideation & 1.42 & 0.74 \\
17 Loss of Interest & 1.55 & 0.81 \\
18 Loss of Energy & 1.88 & 0.90 \\
19 Loss of Pleasure & 1.52 & 0.71 \\
20 Loss of Libido & 1.71 & 0.93 \\
21 Psychomotor Retardation & 1.45 & 0.80 \\
22 Psychomotor Agitation & 1.73 & 0.90 \\
23 Somatic Complaints & 1.99 & 0.80 \\
24 Sympathetic Arousal & 1.78 & 0.71 \\
25 Panic/Phobia & 1.73 & 0.86 \\
26 Gastrointestinal Symptoms & 1.64 & 0.81 \\
28 Interpersonal Sensitivity & & 0.98 \\
\hline
\end{tabular}




\section{Inflammation Biomarkers}

Plasma blood samples were used to assess systemic baseline levels of CRP, IL-6 and TNF- $\alpha$ (details see Supplementary Materials). Markers were selected because they are most commonly studied.

\section{Covariates}

Choice of demographic, lifestyle, and chronic disease-related covariates was based on previous research (Duivis et al. 2013; Haapakoski et al. 2015a). We included age; sex; alcohol intake measured with the Alcohol Use Disorders Identification Test (AUDIT; Saunders, Aasland, Babor, de la Fuente, \& Grant, 1993); smoking status assessed via self-report (never smoker, former smoker, current smoker); BMI; general disease burden measured by the number of chronic diseases currently being treated; and physical activity measured with the International Physical Activity Questionnaire (IPAQ; Craig et al., 2003) in minutes of exercise per week corrected for the amount of energy that a given activity required (MET minutes).

\section{Statistical Analyses and Procedure}

We used the statistical software R (version 3.4.4) to carry out the statistical analyses. Skewed distributions of CRP, IL-6, TNF- $\alpha$, alcohol, exercise, and IDS total score were normalized using the nonparanormal transformation (Liu et al. 2009). The R code to reproduce all analyses is available in the Supplementary Materials; we also provide all model output such as adjacency to make the analyses reproducible.

\section{Network Estimation}

We estimated seven network models (see Table 2 for an overview), from simple to more complicated models.

We estimated unique relations among symptoms, markers, and covariates. In network models, variables are considered "nodes", and "edges" between nodes are conditional dependence relations that can be understood as partial correlations. Given that our data consisted of categorical, ordinal, continuous, and count variables, we estimated Mixed Graphical Models (MGMs) with the R-package $m g m$ (Haslbeck \& Waldrop, 2019). To avoid false positive findings, $m g m$ uses the least absolute shrinkage and selection operator (LASSO, Tibshirani, 1996), leading to a sparse network structure. The LASSO shrinks all edgeweights towards zero and sets small weights to exactly zero. The strength of the penalty is controlled by a parameter lambda, which we selected using the Extended Bayesian Information Criterion (EBIC; Foygel \& Drton, 2010). For a tutorial paper on regularized network models, see Epskamp \& Fried (2018). The EBIC itself has a tuning parameter gamma, which we set to 0 for the main models in the paper (see Supplementary Materials for a detailed rationale). As recommended in recent literature, we 
also estimated the final model without any regularization (Williams et al. 2019) whilst still controlling for the false positive rate.

Table 2. Overview of the seven network models

\begin{tabular}{llll}
\hline Network & Depression variables & Inflammatory markers & Covariates \\
\hline $1 \mathrm{a}$ & IDS total score & CRP, IL-6, TNF- $\alpha$ & -- \\
$1 \mathrm{~b}$ & IDS total score & CRP, IL-6, TNF- $\alpha$ & all* \\
$2 \mathrm{a} * *$ & 9 DSM-5 MDD criteria & CRP & sex and age \\
$2 \mathrm{~b}$ & 9 DSM-5 MDD criteria & CRP & all \\
$3 \mathrm{a}$ & 28 IDS symptoms & CRP, IL-6, TNF- $\alpha$ & -- \\
$3 \mathrm{~b}$ & 28 IDS symptoms & CRP, IL-6, TNF- $\alpha$ & all \\
$4 * * *$ & 28 IDS symptoms & CRP, IL-6, TNF- $\alpha$ & all \\
\hline
\end{tabular}

Note: *all = sex, age, alcohol, smoking, chronic diseases, BMI, exercise. ${ }^{* *}$ Conceptual replication of the study by Jokela et al. (2016).*** Model 4 equals model 3b, with the only difference that model $3 b$ is regularized (which puts small coefficients to exact zero) and leads to a much more conservative estimate of relations, whereas model 4 only controls for multiple testing.

We also estimated node predictability, which quantifies how well a node can be predicted by nodes it shares an edge with. This can be interpreted akin to $\mathrm{R}^{2}$ (Haslbeck \& Fried 2017; Haslbeck \& Waldorp 2017).

We used the R-package qgraph (Epskamp et al. 2012) to visualize the network structures. Blue edges represent positive conditional dependence relations among variables, red edges depict negative relations. We used bootstrapping routines implemented in the package bootnet (Epskamp et al. 2017)to gain information on the precision of parameter estimates (see Supplementary Materials).

\section{Results}

\section{Sample Characteristics}

The final sample $(\mathrm{n}=2321)$ included 808 men $(34.8 \%)$ and 1513 women $(65.2 \%)$. Mean age was 42.9 years $(\mathrm{SD}=12.9)$ for men and 40.5 years for women $(\mathrm{SD}=12.9)$; age range was $18-65$. IDS scores ranged from $0-69$. Half of all participants were not or only mildly depressed. Mean BMI was 25.25 $(\mathrm{SD}=4.6) .870$ participants were current smokers (37.7\%); 780 used to smoke (33.4\%); and 671 had never been regular smokers (28.8\%). The mean score on the AUDIT was $4.9(\mathrm{SD}=4.7)$, indicating that problematic drinking was absent, on average. Mean exercise score amounted to 3685 MET minutes per 
week ( $\mathrm{SD}=3096)$. Finally, 75\% of all participants reported treatment for none or one chronic disease; $25 \%$ reported more.

The means and standard deviations of all symptoms are presented in Table 1. Inflammatory markers were inter-related, with correlations of $r=.30$ (CRP and IL6), $r=16$ (IL6 and TNF- $\alpha$ ), and $r=.14$ (CRP and TNF- $\alpha$ ), which is consistent with different biological functions among markers (Del Giudice \& Gangestad 2018). In the remaining analyses, sex is coded as $m e n=0$ and women $=1$; a positive association between e.g. depression and sex therefore implies that women scored higher on depression.

\section{Depression sum-score model}

We report edge weights and predictability values that were most relevant to our research questions. Unless stated otherwise, edge weights represent positive relationships.

Figure 1 shows the relationship between IDS total score and inflammatory markers without covariates (1a) and with covariates (1b). In network 1a, the IDS total score was related to CRP and IL-6. Inflammatory markers were related amongst each other, with the highest regularized partial correlation between CRP and IL-6. IL-6 and CRP yielded the highest predictability estimates (10.9\% and 10.2\%, respectively). Predictability of the IDS sum score was $1.4 \%$, indicating that it shared little variance with the other variables.

When corrected for covariates (network 1b), the relationship between IDS total score and IL-6 was attenuated, and the link with CRP disappeared. Instead, the IDS total score shared an edge with all covariates, strongest of which were those with chronic diseases and smoking. The IDS total score was negatively related to exercise; CRP was positively related with sex, IL-6 and TNF- $\alpha$ negatively; CRP and IL-6 were related to BMI. BMI had the highest predictability value (25.7\%); followed by $21.4 \%$ for CRP, $15 \%$ for IL-6, and $4.8 \%$ for TNF- $\alpha$. Predictability for the IDS total score increased to $7.9 \%$.

\section{Replication and extension of Jokela et al. (2016)}

Figure 2 depicts results that build on and extend the findings of Jokela et al. (2016). Network 2a shows the relationship between DSM-5 MDD criteria and CRP with sex and age as covariates. CRP was associated with 'sleep problems', 'energy level', and 'appetite/weight' - the same symptoms identified by that Jokela et al. (2016) - and further with age and sex. Highest predictability scores were observed for 'interest/pleasure' (58.6\%), 'sad mood' (54.4\%), and 'energy level' (51.1\%). CRP predictability was $3.4 \%$. When corrected for the influence of additional covariates, CRP shared an edge with 'sleep problems' and 'energy level' (2b). Moreover, CRP showed a strong edge with BMI as well as connections with sex and smoking. Age and chronic diseases were correlated. Highest predictability values were observed for 'interest/pleasure' (58.5\%), 'sad mood' (54.3\%), and 'level of energy' (51.8\%). CRP predictability increased to $17.3 \%$; predictability for BMI was $26.3 \%$. 

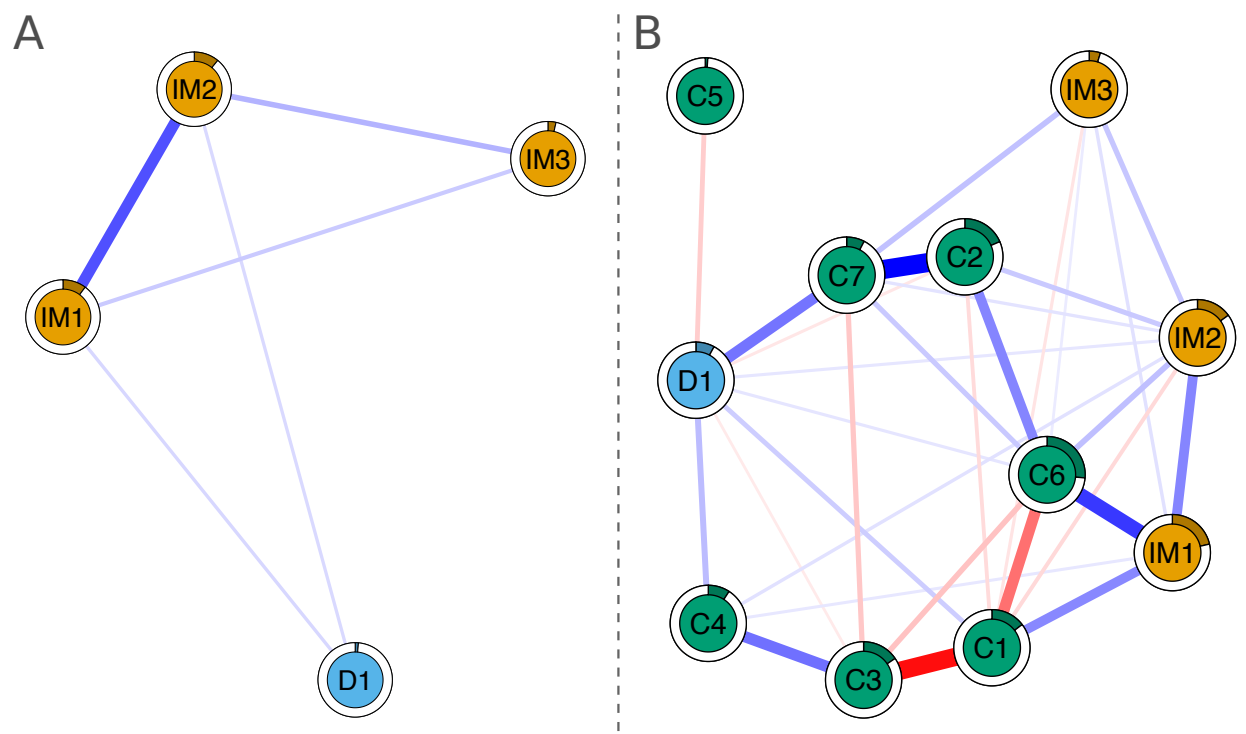
Inflammatory Markers
- IM1: CRP
- IM2: IL-6
- IM3: TNF-alpha
Depression Total
- D1: IDS Total
Covariates
- C1: Sex
- C2: Age
- C3: Alcohol
- C4: Smoking
- C5: Exercise
- C6: BMI
- C7: Chronic Diseases

Figure 1: Network displaying the relationship between IDS total score and inflammatory markers before (panel A) and after controlling for covariates (panel B). Blue edges constitute positive partial correlations between variables, red edges constitute negative partial correlations; rings around nodes convey variance in a given variable with shadowed parts displaying that part of the variance in each node that is explained by nodes that connect with it.
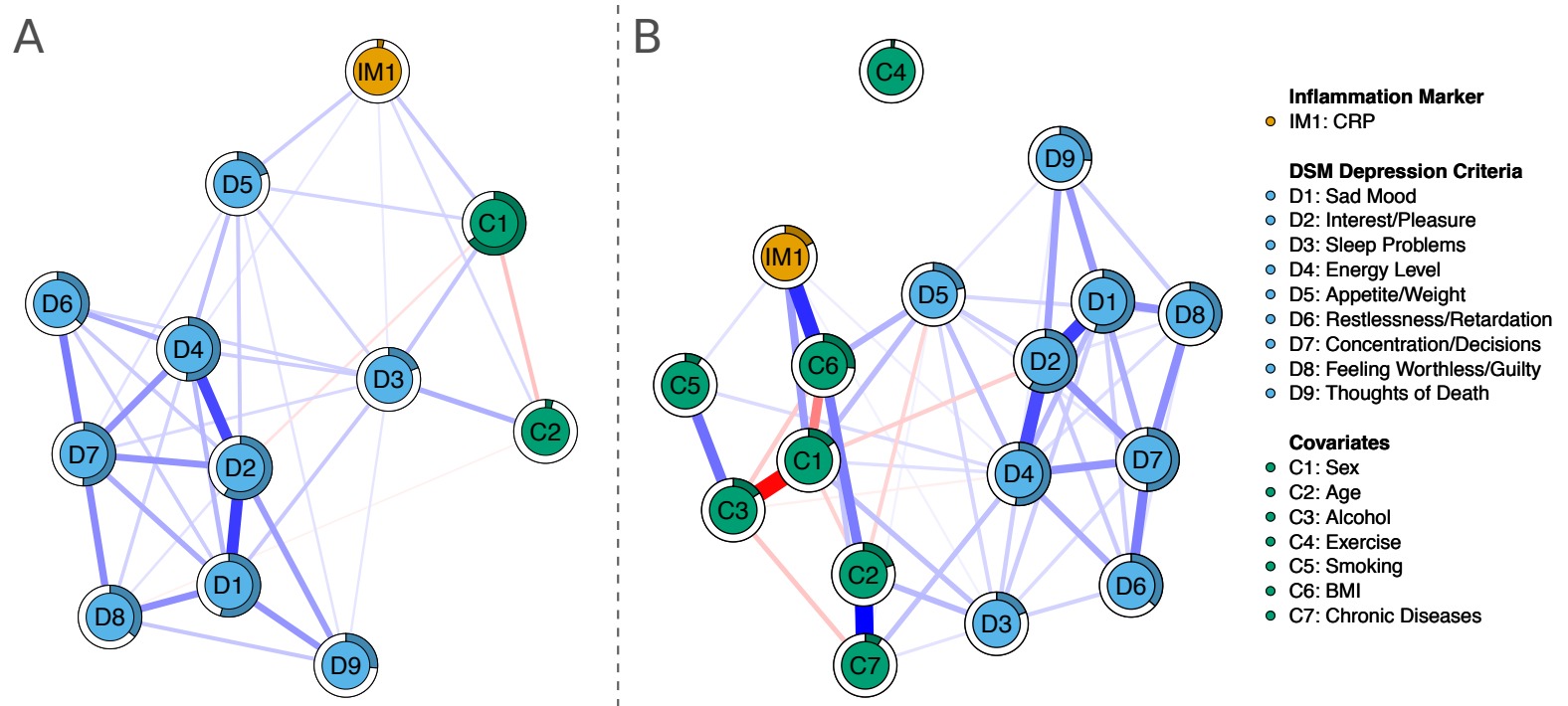

Figure 2: Panel A: Network displaying the results of the conceptual replication of the study by Jokela et al. (2016), featuring DSM-5 MDD criteria, CRP, and covariates. Panel B: Extension of the original study, excluding 5 additional covariates. Blue edges constitute positive partial correlations between variables, red edges constitute negative partial correlations; rings around nodes convey variance in a given variable with shadowed parts displaying that part of the variance in each node that is explained by nodes that connect with it. 


\section{Regularized full model}

Figure 3 displays the relationship between 28 depressive symptoms and inflammatory markers without covariates (3a) and with covariates (3b). In network 3a, CRP and IL-6 were associated with 'aches and pain'. Symptoms tended have higher predictability values (e.g. 'sad mood', 65.3\%; 'interest', 55.2\%; 'energy level', 59.4\%) than inflammatory markers (CRP, 9.2\%; IL-6, 10.8\%; TNF- $\alpha, 3 \%$ ).

When corrected for covariates ( $3 \mathrm{~b}$ ), no single edge emerged between markers and depressive symptoms emerged, while connections among inflammatory markers remained robust. CRP was connected with sex and BMI; IL-6 shared an edge with age and BMI; TNF- $\alpha$ was connected with chronic diseases. Predictability was $19.8 \%$ for CRP, $11.9 \%$ for IL- 6 , and $4.4 \%$ for TNF- $\alpha$. Predictability of depressive symptoms varied considerably, from $9.1 \%$ for 'sleeping too much' to $65.3 \%$ for 'feeling sad'. Predictability values were $26.6 \%$ for BMI, and $17.2 \%$ for chronic diseases.
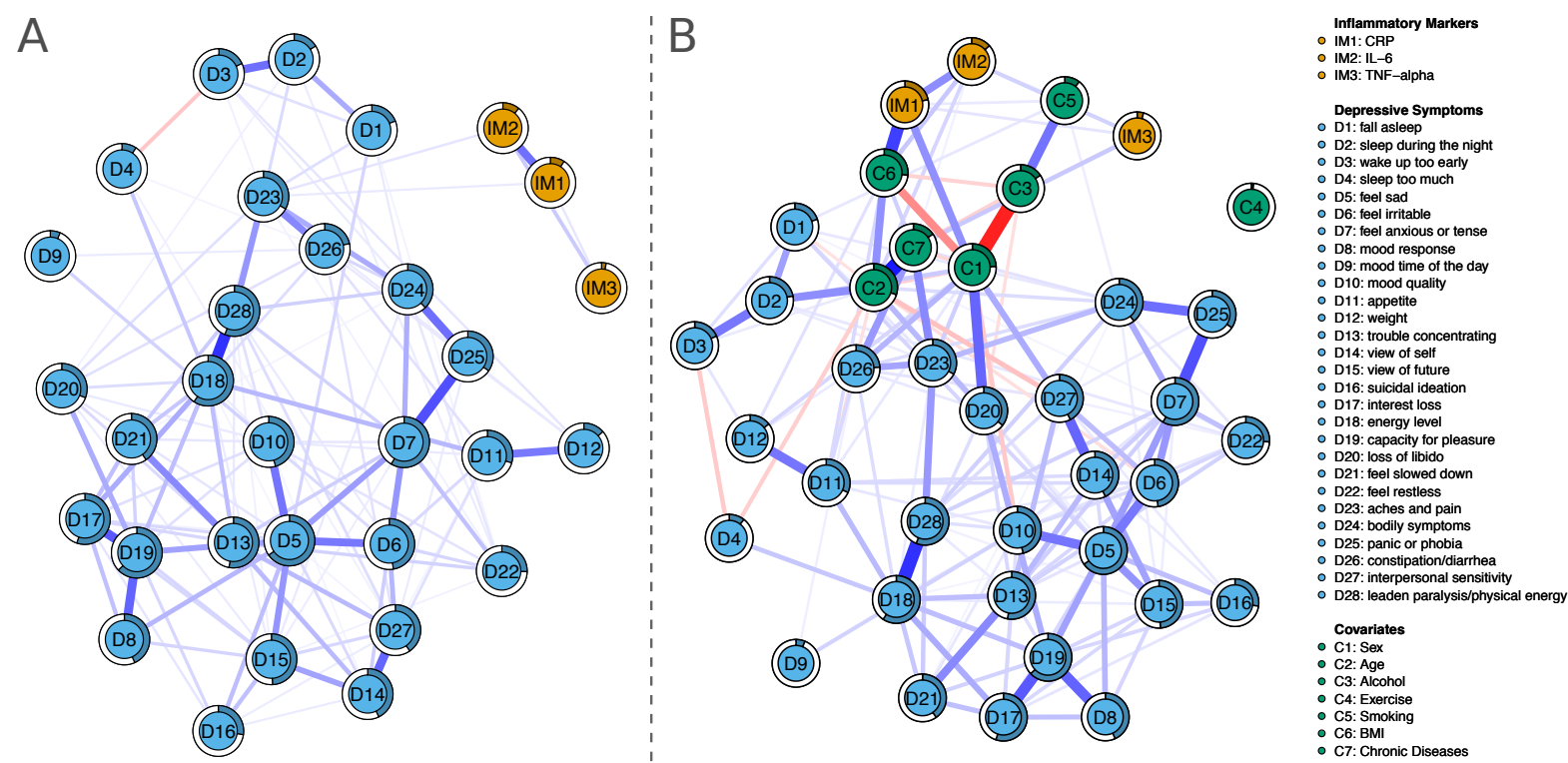

Figure 3: Network displaying the relationship between depressive symptoms and inflammatory markers before (panel A) and after controlling for covariates (panel B). Blue edges constitute positive partial correlations between variables, red edges constitute negative partial correlations; rings around nodes convey variance in a given variable with shadowed parts displaying that part of the variance in each node that is explained by nodes that connect with it.

\section{Non-regularized full model}

We re-estimated the final network $3 \mathrm{~b}$ without regularization while still controlling the false positive rate. This led to a sparse network ( $40.3 \%$ of all edges were exact zero), for which numerous symptom-marker relations emerged (see Supplementary Materials for details). Four symptoms were consistently related 
connected to all three inflammatory markers: 'trouble falling asleep', 'sleep too much', 'aches and pain', and 'irritability'. All except one relationship ('irritability' with IL-6) were positive.
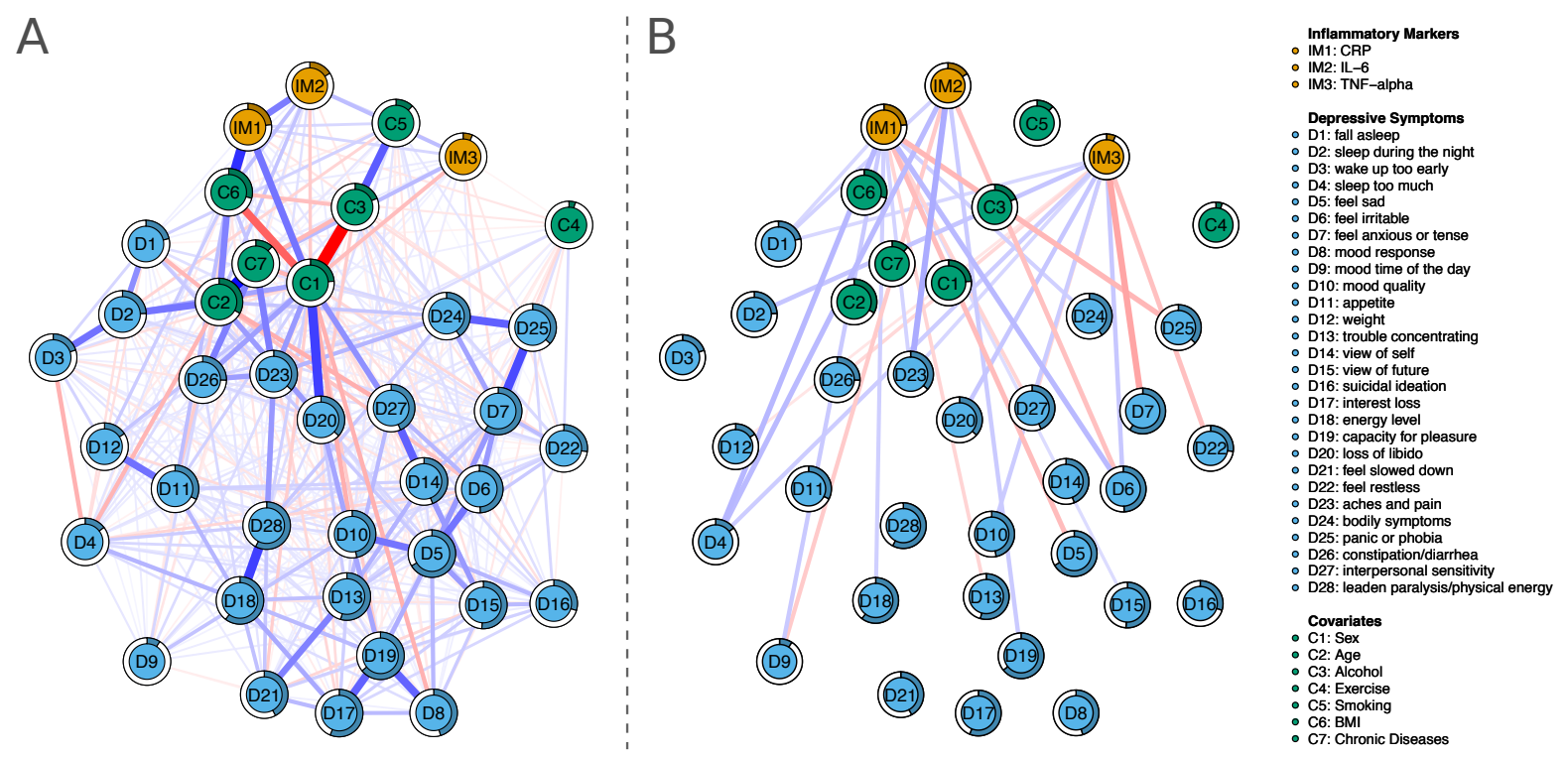

Figure 4: Panel A: Network displaying a less conservative estimation of network $3 b$ containing all variables, without regularization but controlling for multiple testing. Panel B: The same network as in Panel A, except that we only display and zoom in on relations between markers and symptoms to facilitate interpretation.

\section{Sensitivity and Stability Analyses}

In the results, edges between symptoms and markers disappeared in two cases when entering more variables: $2 b$ featured two symptom-marker edges (CRP with 'energy level' \& 'sleep problems'), $3 b$ featured none. Similarly, 3a contained two symptom-marker edges (CRP and IL-6 with 'aches and pain'), which disappeared in $3 \mathrm{~b}$ upon adding covariates. We conducted sensitivity analyses (see Supplementary Materials) to investigate whether these differences could be explained by power differences: $3 b$ had many more parameters (703) than $3 a(465)$ or $2 b(136)$, and therefore less power to detect very small relationships with equal sample size. Our analyses revealed that symptom-marker edges from $2 b$ would not be detected anymore in a network the size of $3 b$ due to lower power, but symptom-marker relations in $3 \mathrm{a}$ would be detected in a network the size of $3 \mathrm{~b}$.

Sensitivity analyses also indicated that all network models re-estimated with a more conservative gamma of 0.25 were identical or nearly identical to the main models (all correlations of adjacency matrices $r>0.99$ ). For $3 a$, 'aches and pains' were no longer associated with either CRP or IL-6.

Stability analyses in which we bootstrapped all models 500 times showed that some edges were estimated reliably (i.e. they were included in all or nearly 500 bootstrapped samples), but there also was considerable variability in the edge parameters across the bootstrapped models. Individual edges and their rank order should be interpreted with care. 


\section{Discussion}

Contrasting prior research based on sum-scores and diagnoses that have yielded inconsistent results regarding the relationship between depression and inflammation, we explored links between individual depressive symptoms, inflammatory markers, and demographic-, lifestyle-, and disease-related covariates in several consecutive models.

A sum-score of depression was linked with IL-6 and CRP in an unadjusted model (1a). When corrected for demographic, lifestyle, and chronic disease-related covariates, the link with IL-6 was greatly reduced, and the relationship with CRP disappeared (1b). Instead, the depression total score shared edges with demographic, lifestyle, and disease-related covariates. Markers, especially CRP, were associated with BMI and sex. In a conceptual replication of Jokela et al. (2016), results closely resembled the original study: sleep problems, energy level, and weight/appetite changes were associated with CRP (2a). When we additionally included BMI, exercise, alcohol, smoking, and chronic diseases as lifestyle and disease-related covariates (2b), only sleep problems, and energy level remained associated with CRP. Moreover, strong edges emerged between CRP and BMI, as well as between CRP and sex. When relationships between inflammatory markers and 28 depressive symptoms were investigated (3a), markers were only associated with aches and pain but no other depressive symptoms. Upon adding lifestyle covariates to the final model (3b), markers and symptoms were unrelated. A strong edge was present between CRP and BMI, while CRP and sex, IL-6 and BMI, and IL-6 and age shared somewhat smaller links. Although markers were related with each other across all models, TNF- $\alpha$ was the least connected marker. When repeating model $3 b$ without regularization while controlling for multiple testing, three symptoms were consistently and positively connected to all three inflammatory markers: trouble falling asleep, hypersomnia, and aches and pain. Irritability showed two positive (CRP, TNF- $\alpha$ ) and one negative (IL-6) association.

Overall, the most likely symptoms to share unique associations with inflammatory markers, based on our and previous cross-sectional work, are: sleep problems, including insomnia and hypersomnia (identified by models 2a, 2b, 3b without regularization, Jokela et al. 2016; White et al. 2017); energy level (models 2a, 2b, Jokela et al. 2016, White et al. 2017); appetite/weight changes (model 2a, Jokela et al. 2016, Lamers et al. 2017); aches and pains (models 3a, 3b without regularization); and irritability (model $3 \mathrm{~b}$ without regularization, including both positive and negative relations).

In all models, BMI was strongly associated with CRP, but weight change and appetite change were not ( $3 a$ and $3 b$ ). One explanation is the comparably low power to detect very small effects, although our sensitivity analyses showed that this explanation is unlikely. It is more likely that potential associations between weight/appetite change and markers were obscured because the symptoms were compound items representing both weight and appetite increases and decreases. Given that CRP is released in adipose tissue (You \& Nicklas 2006; de Heredia et al. 2012), we would expect specifically increases in 
appetite and weight to associate with CRP. Lamers et al. (2017) separated out appetite increase vs decrease, based on CIDI symptoms that allow such a disaggregation, unlike the IDS symptoms used for the present report, and indeed found that increased appetite was related to inflammation, specifically $\mathrm{CRP}$ and TNF- $\alpha$.

Our results show that relationships between depression and inflammation are strongly attenuated after BMI adjustment. This is consistent with some studies in the literature (Elovainio et al. 2009; Liu et al. 2014), but contrasts with other results of more robust associations (Haapakoski et al. 2015b) (however, note that Haapakoski et al. investigated MDD, not depression severity). In addition to the role as inflammatory markers, CRP and IL-6 are synthesized in response to factors emitted by adipose tissue. People with more body fat have higher levels of circulating CRP and IL-6 (You \& Nicklas 2006; de Heredia et al. 2012) which offers one explanation for the strong relation between markers and BMI scores. When considering that adipose tissue produces a significant part of CRP and IL-6, the question arises whether it is sufficient to account for this fact merely by adjusting for BMI as a covariate. It could be that weight represents a major explanatory factor that accounts for the link between depression and inflammation, and that inflammation can occur in depressed patients because certain depressive symptoms emerge as a result from a shared pathophysiology with obesity and metabolic conditions (Lamers et al. 2017; Milaneschi et al. 2018). Future studies may benefit from more closely investigating weight changes and obesity, given that waist circumference and waist-to-hip ratio have been shown to relate to CRP (Choi et al. 2013). In addition to that, it may be helpful to include information about dietary patterns as numerous studies have shown that diet links with levels of systemic inflammation (Slyepchenko et al., 2017; for an overview see Berk et al. 2013). Finally, objective assessment of physical activity may increase insight on top of self-report exercise questionnaires such as the IPAQ. Multiple studies have shown that both acute and regular exercise involve differential release of substances that are also active during inflammation. For example, regular exercise has been shown to down-regulate levels of CRP and IL-6 (Zhou et al. 2010; Hayashino et al. 2014).

It is worth noting that there are numerous other factors that influence inflammation processes, such as physical activity (Zhou et al. 2010; Hayashino et al. 2014) or hormonal changes for women in relation with the menstrual cycle, hormonal contraceptives, or menopause (Vogelzangs et al. 2012). Studies examining inflammation as a potential contributor to depression will benefit from taking these potential explanatory variables into account. This would further address one of the major gaps in the literature (O'Connor et al. 2009; Köhler et al. 2017) that we aimed to address here, i.e. that the inconsistent results reported in previous investigations likely occurred at least in part due to the fact that studies varied in the number and nature of included covariates. Future analyses may also benefit from separately analyzing female and male participants, which was not possible due to power considerations in the current study. 
Overall, the relationship between depression, inflammation, and covariates is likely highly multivariate and multicausal and warrants further investigation. This includes the possibility of reverse causation where depression is not a consequence, but the cause of higher levels of inflammation. For instance, depression as stressor could potentially lead to changes in lifestyle factors such a reduced activity and a poorer diet, which may in turn increase adiposity and thus inflammatory markers.

\section{Limitations and Future Research}

The present paper goes beyond existing literature in several aspects. We used a large sample of 2321 participants along the whole continuum of depression severity; examined relations between different operationalizations of depression severity, 3 inflammatory markers, and 7 covariates; tested the relationships between markers and MDD in various stages of complexity; tested the impact of covariates on associations by estimating models with and without covariates; used network analysis specifically developed for uncovering unique shared associations in highly multivariate data; and provide the first conceptual replication of a symptom-marker study (Jokela et al. 2016) in this emerging field.

Nevertheless, results need to be interpreted with caution. First, we used an observational, cross-sectional design. Cause and effect cannot be established, and conclusions about the direction of a possible relationship between depression, inflammation, and covariates cannot be drawn. Longitudinal followup work should investigate whether the candidate symptoms identified here are predictive of or predicted by inflammatory markers (Smith et al. 2018). Second, there is some evidence that inflammatory markers are related to antidepressant medication (Hiles et al. 2012; White et al. 2017). We did not control for different types of medication because subgrouping would have created insufficient statistical power for network estimation, and future studies should consider studying the link between depression and inflammation in participant clusters grouped by type of medication. There are numerous other covariates that might be related to inflammation (O'Connor et al. 2009; Smith et al. 2018), and numerous further inflammatory markers, which should be studied in the future. Third, we excluded participants with CRP levels above $10 \mathrm{mg} / \mathrm{L}$ to avoid bias due to acute infection, which does not necessarily remove all individuals with minor acute illnesses that could influence both depressive symptoms and inflammatory markers. Fourth, many different depression scales exist, and these scales differ considerably in symptom content (Santor et al. 2006; Fried 2017). We used a comprehensive scale with as many symptoms as possible, but it is an open question if our findings will replicate in a different set of symptoms. This relates to the challenge discussed above that weight and appetite changes could not be disaggregated in the current study, which should be done in the future. Finally, more general challenges to network psychometrics in psychopathology research are presented in detail elsewhere (Fried \& Cramer 2017; Guloksuz et al. 2017). 


\section{Conclusion}

Despite substantial efforts to effectively investigate depression etiology, understanding of this debilitating disorder is limited and research investigating inflammation as a core etiological factor has produced inconsistent results. We aimed to contribute to this ongoing debate by approaching the link between depression and inflammation from a different angle via embracing the potential complexity of the depression-inflammation link. We hope that our results may ultimately help disentangle the role that inflammation may play in the development and course of depression.

\section{Acknowledgements}

The infrastructure for the NESDA study (www.nesda.nl) is funded through the Geestkracht program of the Netherlands Organisation for Health Research and Development (ZonMw, grant number 10-0001002) and financial contributions by participating universities and mental health care organizations (VU University Medical Center, GGZ inGeest, Leiden University Medical Center, Leiden University, GGZ Rivierduinen, University Medical Center Groningen, University of Groningen, Lentis, GGZ Friesland, GGZ Drenthe, Rob Giel Onderzoekscentrum). Assaying of inflammatory markers was supported by the Neuroscience Campus Amsterdam.

During writing this paper, EIF was in part funded by the ERC Consolidator Grant no. 647209.

\section{Data access}

According to European law (GDPR), data containing potentially identifying or sensitive patient information are restricted; our data involving clinical participants are not freely available in the manuscript, supplemental files, or in a public repository. Data access can be requested via the NESDA Data Access Committee (nesda@ggzingeest.nl).

\section{Declaration of interests}

None. 


\section{References}

Berk M, Williams L, Jacka F, O’Neil A, Pasco J, Moylan S, Allen N, Stuart A, Hayley A, Byrne M, Maes M (2013). So depression is an inflammatory disease, but where does the inflammation come from? BMC Medicine 11, 1-16.

Bockting CL, Hollon SD, Jarrett RB, Kuyken W, Dobson K (2015). A lifetime approach to major depressive disorder: The contributions of psychological interventions in preventing relapse and recurrence. Clinical Psychology Review 41, 16-26.

Choi J, Joseph L, Pilote L (2013). Obesity and C-reactive protein in various populations: a systematic review and meta-analysis. Obesity Reviews 14, 232-244.

Chu AL, Stochl J, Lewis G, Zammit S, Jones PB, Khandaker GM (2019). Longitudinal association between inflammatory markers and specific symptoms of depression in a prospective birth cohort. . Elsevier Inc. Brain, Behavior, and Immunity 76, 74-81.

CRAIG CL, MARSHALL AL, SJ??STR??M M, BAUMAN AE, BOOTH ML, AINSWORTH BE, PRATT M, EKELUND U, YNGVE A, SALLIS JF, OJA P (2003). International Physical Activity Questionnaire: 12-Country Reliability and Validity. Medicine \& Science in Sports \& Exercise 35, 1381-1395.

Dantzer R, O'Connor JC, Freund GG, Johnson RW, Kelley KW (2008). From inflammation to sickness and depression: when the immune system subjugates the brain. Nature reviews. Neuroscience 9, 46-56.

Duivis HE, Vogelzangs N, Kupper N, de Jonge P, Penninx BWJH (2013). Differential association of somatic and cognitive symptoms of depression and anxiety with inflammation: findings from the Netherlands Study of Depression and Anxiety (NESDA). . Elsevier Ltd Psychoneuroendocrinology 38, 1573-85.

Dunn BD (2012). Helping Depressed Clients Reconnect to Positive Emotion Experience: Current Insights and Future Directions. Clinical Psychology \& Psychotherapy 19, 326-340.

Elovainio M, Aalto A-M, Kivimäki M, Pirkola S, Sundvall J, Lönnqvist J, Reunanen A (2009). Depression and C-Reactive Protein: Population-Based Health 2000 Study. Psychosomatic Medicine 71, 423-430.

Epskamp S, Borsboom D, Fried EI (2017). Estimating Psychological Networks and their Accuracy: A Tutorial Paper. . Behavior Research Methods Behavior Research Methods, 1-34.

Epskamp S, Cramer AOJ, Waldrop LJ, Schmittmann VD, Borsboom D (2012). qgraph. Network visualizations of relationships in psychometric data $\mathbf{4 8}, 1-18$.

Epskamp S, Fried EI (2018). A Tutorial on Regularized Partial Correlation Networks A Tutorial on Regularized Partial Correlation Networks. Psychological Methods

Foygel R, Drton M (2010). Extended Bayesian Information Criteria for Gaussian Graphical Models. In Advances in Neural Information Processing Systems 23, pp604-612.

Fried EI (2017). The 52 symptoms of major depression: Lack of content overlap among seven common depression scales. . Elsevier Journal of Affective Disorders 208, 191-197.

Fried EI, Cramer AOJ (2017). Moving forward: challenges and directions for psychopathological network theory and methodology. Perspectives on Psychological Science, 1-22.

Fried EI, Nesse RM (2014). The Impact of Individual Depressive Symptoms on Impairment of Psychosocial Functioning. Ed. Q Gong PLoS ONE 9, e90311. 
Fried EI, Nesse RM (2015). Depression sum-scores don’t add up: why analyzing specific depression symptoms is essential. BMC Medicine 13, 1-11.

Fried EI, Nesse RM, Zivin K, Guille C, Sen S (2014). Depression is more than the sum score of its parts: individual DSM symptoms have different risk factors. Psychological medicine 44, 2067-2076.

Del Giudice M, Gangestad SW (2018). Rethinking IL-6 and CRP: Why they are more than inflammatory biomarkers, and why it matters. . Elsevier Inc. Brain, Behavior, and Immunity 70, 6175 .

Guloksuz S, Pries L-K, Van Os J (2017). Application of network methods for understanding mental disorders: pitfalls and promise. Psychological medicine, 1-10.

Haapakoski R, Mathieu J, Ebmeier KP, Alenius H, Kivimäki M (2015a). Cumulative metaanalysis of interleukins 6 and $1 \beta$, tumour necrosis factor $\alpha$ and C-reactive protein in patients with major depressive disorder. . Elsevier Inc. Brain, Behavior, and Immunity 49, 206-215.

Haapakoski R, Mathieu J, Ebmeier KP, Alenius H, Kivimäki M (2015b). Cumulative metaanalysis of interleukins 6 and $1 \beta$, tumour necrosis factor $\alpha$ and C-reactive protein in patients with major depressive disorder. Brain, Behavior, and Immunity 49, 206-215.

Haroon E, Raison CL, Miller AH (2012). Psychoneuroimmunology Meets Neuropsychopharmacology: Translational Implications of the Impact of Inflammation on Behavior. Neuropsychopharmacology 37, 137-162.

Haslbeck J, Fried EI (2017). How Predictable are Symptoms in Psychopathological Networks? A Reanalysis of 17 Published Datasets

Haslbeck J, Waldorp L (2017). How well do network models predict future observations? On the importance of predictability in network models. . Behavior Research Methods Behavior Research Methods, 1-17.

Haslbeck J, Waldrop LJ (2019). MGM: Estimating Time-Varying Mixed Graphical Models in HighDimensional Data. Arxiv preprint

Hayashino Y, Jackson JL, Hirata T, Fukumori N, Nakamura F, Fukuhara S, Tsujii S, Ishii H (2014). Effects of exercise on C-reactive protein, inflammatory cytokine and adipokine in patients with type 2 diabetes: A meta-analysis of randomized controlled trials. Metabolism 63, 431-440.

de Heredia FP, Gómez-Martínez S, Marcos A (2012). Obesity, inflammation and the immune system. Proceedings of the Nutrition Society 71, 332-338.

Hieronymus F, Emilsson JF, Nilsson S, Eriksson E (2016). Consistent superiority of selective serotonin reuptake inhibitors over placebo in reducing depressed mood in patients with major depression. . Nature Publishing Group Molecular Psychiatry 21, 523-30.

Hiles S a, Baker AL, de Malmanche T, Attia J (2012). A meta-analysis of differences in IL-6 and IL-10 between people with and without depression: exploring the causes of heterogeneity. . Elsevier Inc. Brain, behavior, and immunity 26, 1180-8.

Horn SR, Long MM, Nelson BW, Allen NB, Fisher PA, Byrne ML (2018). Replication and reproducibility issues in the relationship between C-reactive protein and depression: A systematic review and focused meta-analysis. . Elsevier Brain, Behavior, and Immunity 73, 85-114.

Jokela M, Virtanen M, Batty GD (2016). Inflammation and specific symptoms of depression. JAMA psychiatry 73, 1-6.

Keller MC, Neale MC, Kendler K (2007). Association of different adverse life events with distinct patterns of depressive symptoms. The American journal of psychiatry 164, 1521-9. 
Kendler K (2016). The Phenomenology of Major Depression and the Representativeness and Nature of DSM Criteria. American Journal of Psychiatry, appi.ajp.2016.1.

Köhler CA, Freitas TH, Maes M, de Andrade NQ, Liu CS, Fernandes BS, Stubbs B, Solmi M, Veronese N, Herrmann N, Raison CL, Miller BJ, Lanctôt KL, Carvalho AF (2017). Peripheral cytokine and chemokine alterations in depression: a meta-analysis of 82 studies. Acta Psychiatrica Scandinavica 135, 373-387.

Lamers F, Bot M, Jansen R, Chan M, Cooper J, Bahn S, Penninx B (2016). Serum proteomic profiles of depressive subtypes. Nature Publishing Group $\mathbf{6}$

Lamers F, Milaneschi Y, de Jonge P, Giltay EJ, Penninx BWJH (2017). Metabolic and inflammatory markers: associations with individual depressive symptoms. Psychological Medicine, 111.

Liu H, Lafferty J, Wasserman L (2009). The nonparanormal: semiparametric estimation of high dimensional undirected graphs. The Journal of Machine Learning Research 10, 2295-2328.

Liu Y, Al-Sayegh H, Jabrah R, Wang W, Yan F, Zhang J (2014). Association between C-reactive protein and depression: Modulated by gender and mediated by body weight. . Elsevier Psychiatry Research 219, 103-108.

Milaneschi Y, Simmons WK, van Rossum EFC, Penninx BW (2018). Depression and obesity: evidence of shared biological mechanisms. Molecular Psychiatry

Moriarity DP, Mac Giollabhui N, Ellman LM, Klugman J, Coe CL, Abramson LY, Alloy LB (2019). Inflammatory Proteins Predict Change in Depressive Symptoms in Male and Female Adolescents. Clinical Psychological Science

O'Connor MF, Bower JE, Cho HJ, Creswell JD, Dimitrov S, Hamby ME, Hoyt MA, Martin JL, Robles TF, Sloan EK, Thomas KMS, Irwin MR (2009). To assess, to control, to exclude: Effects of biobehavioral factors on circulating inflammatory markers. . Elsevier Inc. Brain, Behavior, and Immunity 23, 887-897.

Penninx BWJH, Beekman ATF, Smit JH, Zitman FG, Nolen WA, Spinhoven P, Cuijpers P, De Jong PJ, Van Marwijk HWJ, Assendelft WJJ, Van Der Meer K, Verhaak P, Wensing M, De Graaf R, Hoogendijk WJ, Ormel J, Van Dyck R (2008). The Netherlands Study of Depression and Anxiety (NESDA): rationale, objectives and methods. International journal of methods in psychiatric research 17, 121-40.

Rush AJ, Gullion CM, Basco MR, Jarrett RB, Trivedi MH (1996). The Inventory of Depressive Symptomatology (IDS): psychometric properties. Psychological medicine 26, 477-86.

Santor DA, Gregus M, Welch A (2006). Eight Decades of Measurement in Depression. Measurement 4, 135-155.

Saunders JB, Aasland OG, Babor TF, de la Fuente JR, Grant M (1993). Development of the Alcohol Use Disorders Identification Test (AUDIT): WHO Collaborative Project on Early Detection of Persons with Harmful Alcohol Consumption--II. Addiction (Abingdon, England) 88, 791-804.

Slyepchenko A, Maes M, Jacka FN, Köhler CA, Barichello T, McIntyre RS, Berk M, Grande I, Foster JA, Vieta E, Carvalho AF (2017). Gut Microbiota, Bacterial Translocation, and Interactions with Diet: Pathophysiological Links between Major Depressive Disorder and Non-Communicable Medical Comorbidities. Psychotherapy and Psychosomatics 86, 31-46.

Smith KJ, Au B, Ollis L, Schmitz N (2018). The association between C-reactive protein, Interleukin6 and depression among older adults in the community: A systematic review and meta-analysis. . Elsevier Experimental Gerontology 102, 109-132. 
Smith RS (1991). The macrophage theory of depression. Medical hypotheses 35, 298-306.

Stringaris A, Belil PVR, Artiges E, Lemaitre H, Gollier-Briant F, Wolke S, Vulser H, Miranda R, Penttilä J, Struve M, Fadai T, Kappel V, Grimmer Y, Goodman R, Poustka L, Conrod P, Cattrell A, Banaschewski T, Bokde ALW, Bromberg U, Büchel C, Flor H, Frouin V, Gallinat J, Garavan H, Gowland P, Heinz A, Ittermann B, Nees F, Papadopoulos D, Paus T, Smolka MN, Walter H, Whelan R, Martinot JL, Schumann G, Paillère-Martinot ML, Poline JB, Pausova Z, Mann K, Barker GJ, Lawrence C, Loth E, Rietschel M, Robbins TW, Reed L, Williams S, Lourdusamy A, Costafreda S, Cattrell A, Nymberg C, Topper L, Smith L, Havatzias S, Stueber K, Mallik C, Clarke TK, Stacey D, PengWong C, Werts H, Williams S, Andrew C, Desrivieres S, Zewdie S, Häke I, Ivanov N, Klär A, Reuter J, Palafox C, Hohmann C, Schilling C, Lüdemann K, Romanowski A, Ströhle A, Wolff E, Rapp M, Brühl R, Ihlenfeld A, Walaszek B, Schubert F, Connolly C, Jones J, Lalor E, McCabe E, NíShiothcháin A, Spanagel R, Leonardi-Essmann F, Sommer W, Vollstaedt-Klein S, Steiner S, Buehler M, Stolzenburg E, Schmal C, Schirmbeck F, Heym N, Newman C, Huebner T, Ripke S, et al. (2015). The brain s response to reward anticipation and depression in adolescence: Dimensionality, specificity, and longitudinal predictions in a community-based sample. American Journal of Psychiatry 172, 1215-1223.

Tweed DL (1993). Depression-related impairment: estimating concurrent and lingering effects. Psychological Medicine 23, 373-386.

Valkanova V, Ebmeier KP, Allan CL (2013). CRP, IL-6 and depression: a systematic review and meta-analysis of longitudinal studies. . Elsevier Journal of affective disorders 150, 736-44.

Vogelzangs N, Duivis HE, Beekman ATF, Kluft C, Neuteboom J, Hoogendijk W, Smit JH, de Jonge P, Penninx BWJH (2012). Association of depressive disorders, depression characteristics and antidepressant medication with inflammation. . Nature Publishing Group Translational psychiatry $\mathbf{2}$, e79.

White J, Kivimäki M, Jokela M, Batty GD (2017). Association of inflammation with specific symptoms of depression in a general population of older people: The English Longitudinal Study of Ageing. . Elsevier Inc. Brain, Behavior, and Immunity 61, 27-30.

Williams D, Rhemtulla M, Wysocki AC, Rast P (2019). On Non-Regularized Estimation of Psychological Networks. Preprint

You T, Nicklas BJ (2006). Chronic inflammation: role of adipose tissue and modulation by weight loss. Current diabetes reviews 2, 29-37.

Zhou X, Fragala MS, McElhaney JE, Kuchel GA (2010). Conceptual and methodological issues relevant to cytokine and inflammatory marker measurements in clinical research. Current Opinion in Clinical Nutrition and Metabolic Care 13, 541-547. 